\title{
COORDINACIÓN ENTRE DISPOSITIVOS DE EDUCACIÓN Y SALUD MENTAL INFANTO JUVENIL
}

COORDINATION BETWEEN EDUCATION AND MENTAL HEALTH SERVICES FOR CHILDREN AND ADOLESCENTS

\section{RESUMEN}

Se plantea la necesidad del trabajo interdisciplinar y coordinado entre los distintos dispositivos que atienden al niño y al adolescente, ya que aún hoy en día ante los problemas psíquicos, educativos o sociales que se puedan presentar en estas edades, existen diferentes equipos implicados para la atención de los mismos, por lo que es difícil en la práctica trabajar desde una visión integral e integrada, haciéndolo por lo general separadamente.

Partiendo de la necesidad de tener una red amplia y completa de dispositivos de salud mental infanto juvenil, se plantean las distintas áreas de coordinación y diferentes dispositivos para la atención integrada de los niños y adolescentes con trastorno mental grave y con necesidades educativas especiales.

Palabras clave: Trastorno mental grave en niños y adolescentes; trabajo interdisciplinar; dispositivos para atención integrada.

\section{ABSTRACT}

This raises the question of interdisciplinary work and the need for the different devices that help us pay attention to children and teenagers to be coordinated. This is important because still today there are many different teams involved in meeting the psychological, educational and social problems that children of these ages have.

This makes it very difficult to work from an integral and integrated perspective as it is done separatedly. Departing from the fact that we need a wide and complete net of mental health devices addressed to infants and young people, we establish the different areas of coordination and the different devices to give an integral respond to children and adolescents with mental disorders and special educational needs.

Key words: Serious mental disorders in children and adolescents, interdisciplinary work, devices for integrated attention. 


\section{INTRODUCCIÓN}

Durante seis años ha estado funcionando un grupo de trabajo convocado y organizado por la Sección de Salud Mental Infanto-Juvenil de la Asociación Española de Neuropsiquiatría (A.E.N), que ha ido debatiendo y elaborando distintos documentos sobre dispositivos y abordaje de la problemática mental en niños y adolescentes, documentos que han sido publicados posteriormente en la revista de la AEN (1)(2)(3).

Continuando con los planteamientos de estos grupos, se vio la necesidad de plantear el trabajo interdisciplinar y coordinado entre los distintos dispositivos que atienden al niño y al adolescente, de lo cual es fruto este nuevo documento.

La infancia y adolescencia son periodos evolutivos cruciales, cuyo desarrollo armonioso depende de factores biológicos, sociales y educativos, así como familiares y relacionales, aunque la interpretación de la importancia que tiene cada área en la génesis y la influencia en el desarrollo emocional y cognitivo es muy variable y controvertida. Aún hoy en día ante los problemas psíquicos, educativos o sociales que se puedan presentar en estas edades, existen diferentes equipos implicados para la atención de los mismos, por lo que es difícil en la práctica trabajar desde una visión integral e integrada, haciéndolo por lo general separadamente. (4)

Los profesionales que trabajamos en dispositivos sociales, educativos o de salud mental para niños y adolescentes, no podemos olvidar que son personas en desarrollo y por tanto influenciables por el entorno; a su vez la propia problemática mental, así como las dificultades de aprendizaje o sociales, pueden provocar reacciones en este mismo medio que produzcan de nuevo respuestas que aun empeoren más la situación; o bien por el contrario, la respuesta social (de la familia, del barrio, de la escuela...), acepte, contenga o integre al chico con ciertas dificultades o síntomas, favoreciendo su evolución.

Debemos intervenir en esos ámbitos también desde un enfoque psicoterapéutico y educativo amplio, para comprender cómo influyen en un niño o adolescente dado y para intentar favorecer que ese entorno cambie si es necesario, sobre todo facilitando la comprensión de la sintomatología que presenta y ayudando en su resolución.

Partimos pues de la necesidad de una coordinación y un trabajo interdisciplinar entre los distintos dispositivos que intervenimos en la infancia, para lo que planteamos en el presente trabajo los puntos esenciales que a nuestro entender se han de tener en cuenta para crear las bases de esa coordinación y trabajo interdisciplinar.

Inicialmente nos encontramos con dificultades importantes, necesarias de plasmar pues sin su resolución por parte de las autoridades competentes, los esfuerzos voluntaristas que se realicen, como ocurre en la actualidad, van abocados al fracaso o al menos a rendimientos muy pobres en relación con el considerable esfuerzo realizado por los distintos profesionales: 
- Falta de recursos ambulatorios especialmente en salud mental para niños y adolescentes en las diferentes comunidades autónomas y la precariedad de recursos humanos en los existentes, lo que dificulta la posibilidad de coordinación continuada con el resto de equipos de las áreas sanitarias.

- Escasez por parte de sanidad de recursos intermedios para la patología mental grave, lo que favorece que programas y dispositivos desarrollados por parte de educación para el abordaje de estas problemáticas, sean realizados de forma unilateral por lo general y sin coordinación con los recursos de salud mental.

- La tendencia de los organismos competentes, tanto de Educación, Bienestar Social y Sanidad, a poner en marcha programas y dispositivos, sin tener e cuenta la coordinación y trabajo conjunto de los diferentes profesionales.

- Existencia de lenguajes diferentes en los distintos campos de trabajo, para referirnos a una misma problemática, lo que dificulta la unificación de criterios y la toma de decisiones conjuntas 0 al menos consensuadas.

En términos generales y partiendo de lo expuesto anteriormente, insistimos una vez más en la necesidad de tener una red amplia y completa de dispositivos de salud mental infanto juvenil (3), que de cobertura en cada área sanitaria a las distintas problemáticas mentales que surgen en estas edades.

Así mismo es necesario iniciar una coordinación entre los distintos dispositivos que nos posibilite(4):

- Unificar, conciliar o al menos respetar el lenguaje y la filosofía utilizados desde cada campo de trabajo, de tal modo que eso no sea un impedimento para la coordinación.

- Conocer la especificidad de cada equipo, evitando funcionar en redes o actuaciones paralelas poco coordinadas.

- Mejorar los sistemas de derivación de casos y de coordinación.

- Conocer los problemas psicosociales y psicopedagógicos más relevantes en estas edades y que impliquen a los diversos equipos.

- Realizar protocolos de intervención conjunta en aquellas problemáticas que así lo requieran, abarcando también la perspectiva preventiva.

- Comunicar a las autoridades competentes las necesidades que se detecten en materia de prevención, actuaciones conjuntas y de recursos necesarios.

Para ello se propone la creación de una Comisión técnica interprofesional en cada área sanitaria, de forma oficial integrada por profesionales del área, pertenecientes a SMIJ, Educación y Bienestar Social (y en muchos casos Justicia). 


\section{ÁREAS DE COORDINACIÓN Y TRABAJO INTERDISCIPLINAR}

En primer lugar planteamos la necesidad de contar con una política por parte de las Consejerías implicadas (Educación, Sanidad, Bienestar Social y Justicia), de acuerdos de colaboración y funcionamiento interdisciplinar, proponiendo el desarrollo de programas de prevención y de actuación precoz en determinados grupos de riesgo, donde la salud mental no se diluya dentro de esos acuerdos en la sanidad general.

Así mismo es importante que se puedan poner en marcha convenios de colaboración entre las diferentes Consejerías que posibiliten la creación de dispositivos específicos para el abordaje conjunto de problemáticas mentales donde es necesario no solo la coordinación y el trabajo común de los diferentes profesionales, sino la financiación y el diseño común de los mismos (centros psicoeducativos y/o centros terapéuticos de media y larga estancia, centros terapéuticos de día u hospitales de día...)(3).

Por ello propugnamos la necesidad de que funcionen comisiones técnicas interinstitucionales a niveles regionales, que den curso a los convenios necesarios para que todo esto se pueda desarrollar, en estrecha comunicación con la mencionada Comisión técnica interprofesional de área, viendo las necesidades reales que se tienen.

Esta coordinación se completaría con reuniones periódicas de los diferentes equipos en relación a problemas concretos que plantea un niño/ adolescente y que implican a varios profesionales

A un nivel más concreto y en relación con los problemas que el niño/ adolescente puede plantear en periodo escolar, se propone comenzar con las necesidades básicas de esta coordinación entre los diferentes equipos e ir desarrollando conjuntamente los siguientes puntos:

\section{Primera infancia}

- Coordinación con Centro de Desarrollo Infantil y A. Temprana: funcionamiento conjunto con protocolos para la detección y abordaje precoz de problemas de salud mental (especialmente los de mayor gravedad) en niños de 0 a 3 años.

2. Niños en preescolares y ciclo de primaria

- Coordinación con equipos psicopedagógicos del ciclo de infantil y primaría: derivación de casos en ambos sentidos e intervención conjunta a través de protocolos, proponiendo los siguientes:

- Derivación de casos a SMIJ, e intervención coordinada:

- Retrasos psicomotores (lenguaje, simbolización...)

- Trastornos de conducta, hiperactividad... Disarmonías evolutivas.

- Trastornos Generalizados del Desarrollo- Psicosis. 
- Derivación a Equipos psicopedagógicos (casos vistos en SMIJ) y acuerdos de intervención:

- Para valoración psicopedagógica

- Para posible cambio de escolaridad

- Información- apoyo- coordinación a maestros desde SMIJ.

- Intervenciones concretas (logopedia, apoyo...)

- Coordinación con los departamentos de orientación de enseñanza secundaria:

- Actuación conjunta en los casos atendidos en SMIJ de los trastornos de conducta y de fracaso escolar.

3. Niños y adolescentes con trastorno mental grave y con necesidades educativas especiales

Para muchos niños y adolescentes con requerimientos educativos especiales, que además presentan un trastorno mental severo, son necesarios dispositivos con la participación conjunta de Educación y Salud Mental Infanto Juvenil, siendo así mismo importante la coordinación con Bienestar Social para el abordaje de ciertas situaciones familiares, así como para favorecer la integración social de estos niños y adolescentes.

Para estas problemáticas se ve imprescindible crear o adecuar dispositivos nuevos que den cuenta de esta diversidad y aunque se perfila la conveniencia de diferentes recursos, en todos ellos se ve la necesidad de equipos conjuntos que aborden la problemática diversa.

Tanto desde el ámbito clínico, como desde el psicopedagógico, partimos que se tratan de trastornos mentales, donde además de los posibles déficit cognitivos y relacionales, hay alteraciones emocionales y síntomas psiquiátricos, cuyo origen, en la mayor parte de los casos, es multifactorial.

Por ello se plantea, en un primer momento y de forma prioritaria, que la coordinación parta desde la detección precoz, haciendo especial hincapié en el diagnóstico, siendo importante que además del diagnóstico psicopedagógico, esté presente para el nivel educativo el diagnóstico clínico por parte de Salud Mental y viceversa.

Es fundamental resaltar que el pronóstico de estas patologías se ha revelado hoy por hoy difícil y tortuoso se utilice el método que se utilice, por lo que propugnamos partir de las carencias y limitaciones de cada área, siendo conscientes de que el trabajo coordinado y multiprofesional es el que estipulamos más idóneo. Ante un niño con sospecha de padecer una psicosis o TGD, propugnamos además de una colaboración en el diagnóstico, una toma de decisiones colegiadas entre los equipos psicopedagógicos y de salud mental infanto- juveniles implicados, de cara a que dispositivo debe acudir el niño y cómo se haría la coordinación entre ambos. 
Así mismo esto nos plantea la necesidad de tener un espacio de formación continuada común.

Para poder cumplir estos objetivos, se proponen reuniones periódicas, iniciándolas especialmente a principios de curso.

En relación a los dispositivos podemos especificar:

1. Hospital de Día para niños y adolescentes con trastornos mentales graves (incluidos los de tiempo parcial), aunque puede denominarse así mismo en otros ámbitos Centro terapéutico de día(1).

Es un dispositivo asistencial para el tratamiento institucional intensivo, en un medio terapéutico específico, de los trastornos mentales severos que aparezcan en estos periodos de la vida.

El objetivo clínico fundamental con el niño/ adolescente sería el posibilitar el cambio emocional y cognitivo de forma estable, que permita una mejor adaptación a la realidad, así como una mejora de las competencias relacionales y que facilite la reincorporación a la vida en la comunidad, donde podrán continuar el tratamiento ambulatorio oportuno. Así mismo se plantea como esencial el trabajo con la familia, ayudando a contener y elaborara las ansiedades y ambivalencias generadas por la enfermedad y actuando sobre los factores familiares influyentes en la misma.

El tiempo de permanencia semanal en el dispositivo, es variable, estaría en función de la posibilidad o no de integración en los centros escolares de la red educativa de cada área geográfica.

- Indicaciones clínicas:

Dirigido a una población entre 2 y 17 años (ambos inclusive) que presente cuadros psicóticos u otras alteraciones severas que conlleven un trastorno de la organización del psiquismo y de la relación del niño/ adolescente con su mundo circundante y que impidan su adaptación al medio familiar, social, escolar y /o profesional. Se incluyen:

- Trastornos generalizados del desarrollo.

- Esquizofrenia, Trastorno Esquizotípico.

- Trastornos del Humor.

- Trastornos neuróticos severos.

- Trastornos de personalidad.

- Disarmonías evolutivas severas.

- Trastornos graves de la alimentación.

Debido a la gran disparidad de las edades, se hace necesario especificar claramente tres espacios diferenciados dependiendo de la edad:

- De 2 a 6-7 años.

- De 6-7 años a 12.

- De 12-13 a 17 años inclusive. 
- Recursos humanos

Se parte de la necesidad de equipos multidisciplinares, donde se utilicen técnicas psicoterapéuticas para los trastornos emocionales, junto con trabajo pedagógico necesario para el desarrollo cognitivo y emocional. Se estipula:

- Psiquiatra de niños y adolescentes.

- Psicólogo clínico.

- Logopeda (en el grupo de los pequeños).

- Psicomotricista (pudiendo ser asumida esta función por otro miembro del equipo con formación específica).

- Personal de enfermería y monitores /as.

- Trabajador Social.

- Terapeuta ocupacional.

- Maestro /a especializado en Pedagogía terapéutica.

Ya hay en funcionamiento este tipo de dispositivos en algunas comunidades autónomas, como Navarra, País Vasco(5), Madrid (6) y Cataluña, en las que existen acuerdos de colaboración entre las instituciones implicadas.

2. Aulas específicas para Trastornos Generalizados del Desarrollo (TGD). Este dispositivo desarrollado en la comunidad de Madrid, depende de Educación y es una nueva alternativa que favorece la integración y el abordaje psicopedagógico en la escuela. Es para niños de entre 3 y 12 años con TGD. Se caracteriza por:

- Es un aula especial dentro de un centro de educación ordinario.

- El niño está matriculado en su aula de referencia y combina la escolaridad dentro del mismo colegio.

- Cuenta con un auxiliar y con un especialista en audición y lenguaje (AL) o un profesor de pedagogía terapéutica (PT).

- Para atender este dispositivo, existe un equipo psicopedagógico específico, formado en TGD.

Se propugna la intervención coordinada con el personal educativo, de los profesionales de SMIJ en estas aulas, coordinándose con el personal educativo, que aportarían la comprensión de los conflictos emocionales que estos niños presentan y cómo influyen los mismos tanto en su conducta como en su forma de aprender, así como poder realizar intervenciones familiares. Con una filosofía similar existen alternativas educativas en otras comunidades autónomas (7)(8). 
3. Colegios de educación especial

Existen niños y adolescentes que no se amoldan a los anteriores dispositivos, por lo que requieren más específicamente centros de educación especial, propugnando que participen en los mismos profesionales de salud mental. Dentro de esta problemática se incluirían:

- Niños y adolescentes con deficiencia mental que necesitan una escolaridad en colegios de educación especial, porque presentan cuadros de agitación, de autoagresión y heteroagresión, que son de difícil contención en el ámbito escolar con métodos exclusivamente psicopedagógicos, obedeciendo a veces a psicosis injertadas, a psicosis deficitarias, psicosis de tipo orgánico u otros cuadros psiquiátricos graves.

- Niños y adolescentes con trastornos psicóticos o trastornos graves de la personalidad, con deficiencia mental leve o moderada (en ocasiones como síntoma residual de su patología mental de base). Niños y adolescentes con Trastornos Generalizados del Desarrollo y otros cuadros graves psiquiátricos, que por su sintomatología excede la capacidad de tratamiento del Hospital de Día o Centro terapéutico y que así mismo no pueden ser integrados en los colegios normalizados, por lo que deben asistir a colegios de educación especial.

En algunos de estos casos la situación familiar es difícil y complicada por diversos motivos ( en ocasiones provocada o agravada por la propia sintomatología del chico), no pudiendo en muchos momentos dar una cobertura de contención emocional apropiada al mismo, siendo una importante medida terapéutica la separación del niño o adolescente del medio familiar.

Por otra parte muchos de estos niños y adolescentes, aunque evolucionen favorablemente, sufren una descompensación de su cuadro en los periodos vacacionales, siendo importante plantear una continuidad asistencial durante estos periodos.

Para dar cobertura a estas diferentes situaciones se ve necesario la siguiente organización:

- Colegio de educación especial con refuerzo de Salud Mental Infanto Juvenil (SMIJ) (9).

- Dispositivos de internamiento terapéutico y residenciales.

- Internamiento en colegios de educación especial (siendo necesario también el refuerzo de SMIJ)

- Familias terapéuticas con el apoyo de USMIJ, para casos como el apartado anterior, que si se pudieran beneficiar de este dispositivo.

- Colegios de educación especial con cobertura completa vacacional y respiro familiar, debiendo desarrollarse este programa conjuntamente con Bienestar Social. 


\section{BIBLIOGRAFÍA}

1. Jiménez Pascual, A. (coordinadora del grupo trabajo). Hospital de día psiquiátrico para niños y adolescentes. Revista Asociación española de Neuropsiquiatría 2001; vol XXI, (77): pp. 115- 124.

2. Jiménez Pascual, A. (coordinadora del grupo trabajo). Detección y atención precoz de la patología mental en la primera infancia. Revista de la Asociación Española de Neuropsiquiatría 2002; vol XXII, (82): pp. 115- 126.

3. Jiménez Pascual, A. (coordinadora del grupo trabajo). Dispositivos de internamiento terapéutico para patología mental severa de niños y adolescentes. Revista Asociación Española de Neuropsiquiatría 2004; vol, nº, pp

4. Jiménez Pascual, A. Intervenciones psicoterapéuticas en unidades de salud mental infanto juvenil y coordinación con otros dispositivos (en prensa, revista de SEPYPNA).

5. Begoña Solana Azumendi. Lugar de encuentro en la atención a los trastornos mentales severos en la infancia. Revista Norte de Salud Mental 2001; IV, (12): 75-80

6. Orden 992/2002 de 11 de Diciembre. B.O.C.M. Num. 19 (publicado 23 de Enero de 2003).

7. Resolución de 25-07-2001, de la Dirección general de Coordinación y Política educativa. Castilla La Mancha, D.0.C.M. Num. 89 (publicado el 10 de agosto de 2001).

8. Resolución 08-07-2002, de la Dirección de Coordinación y Política Educativa. Castilla La Mancha, D.0.C.M. Num. 88 (publicado el 19 de julio de 2002).

9. Prego Dorca, R. Centro de rehabilitación psico- social infanto- juvenil. Memoria técnica. Fundación Obra san Martín. Colegio de educación especial P. Apolinar (trabajo no publicado).

GRUPO DE TRABAJO

Roque Prego Dorca

Centro Salud Mental Infantil

Santander

Ana-Ma Jiménez Pascual

Unidad S.Mental Infanto-Juvenil

C/ Irlanda 7

13600-Alcazar de S.Juan 
Begoña Solana Azurmendi

Centro de Día educativo terapéutico

Bilbao

Carmen González Noguera

Hosp.Insular de Gran Canarias

Las Palmas de G.Canaria

Elena Cortés Rabal

U.S.M. Las Palmas

Lucía Álvarez Buylla

CSM Ciudad Lineal

Madrid

Teresa Armas Falcón

U.S.M. Lasso Las Palmas

Carmen Otero

Serv . de planificación

Consejería de educación

Comunidad de Madrid

Lourdes Sánchez

Unidad de S.M.I.J. Hospital de Valmes

Sevilla

Carmen Alriols Fornes

Serv. Educación especial y orientación psicopedagógica

Consejería de educación. Comunidad de Madrid

Mercé Gilbert i Clols

Hospital de Día para Adolescentes de Gavá

Barcelona

Ma Angels Horta Guitart

Hospital de Día para Adolescentes de Gavá

Barcelona 
Mateo Martínez

Serv. Educación especial y orientación psicopedagógica

Consejería de educación. Comunidad de Madrid

Rubén Estandia

Centro "Lupasco" Trastornos de personalidad

Olga Ramírez Muñoz

Logopeda. Coordinadora del CDIAT ASPRODIQ

Quintanar de la Orden. Toledo

Paloma San Román

Psiquiatra U.S.M.I.J

Albacete 